\title{
A HIGH-CURRENT DENSITY CONTACT IONIZATION SOURCE FOR HEAVY-ION FUSION *
}

\author{
$\underline{\text { S. MacLaren }}^{\#}$, D. Beck, A. Faltens, W. Ghiorso, E. Henestroza, P. Seidl, Lawrence Berkeley \\ National Laboratory, Berkeley, CA 94720
}

\begin{abstract}
Heavy ion fusion (HIF) sources will be low-emittance, high current (0.5-1A), and suitable for injection into a multiple beam induction linac, where the diameter of each beam aperture at the beginning of the accelerator is 3-6 $\mathrm{cm}$. Practical transport limits involved in matching the beam to the accelerator aperture in a reasonably-sized structure correspond to several $\mathrm{mA} / \mathrm{cm}^{2}$ of space charge limited flow with an injector extraction voltage of 1-2.5 MV. We have built a $\mathrm{J} \approx 8 \mathrm{~mA} / \mathrm{cm}^{2} \mathrm{Cs}^{+}$contact ionization source for a $160 \mathrm{kV}$ diode; this represents a factor of four increase in the $\mathrm{Cs}^{+}$current density over previous sources for HIF. The emitter is sintered iridium, operating at $950<\mathrm{T}<1150{ }^{\circ} \mathrm{C}$. We are experimenting with several methods of alkali feeding capable of maintaining a fraction $(<5 \%)$ of a monolayer coverage on the iridium surface.
\end{abstract}

\section{BACKGROUND}

In developing sources and injectors for a heavy ion fusion driver, high current density is desired because it allows smaller and/or fewer beams and thus a less expensive injector design. Ideally, one desires that the current density be limited by voltage breakdown and beam transport considerations rather than the emission limit of the ion source. Recent designs, which are still evolving, place the desired current density of the ion source at 8 $\mathrm{mA} / \mathrm{cm} 2 \mathrm{Cs}+$ equivalent.

At Lawrence Berkeley National Laboratory, a surface ionization source that achieves > $8 \mathrm{~mA} / \mathrm{cm} 2$ has been built and tested. This particular type of surface ionizer, in which Cs atoms are first applied to and then evaporated from a refractory metal surface, has routinely produced highly uniform ion beams with low emittance. The resulting transverse emittance for an injector with good optics is slightly above the lower limit established by the temperature of the emitting surface $(\sim 0.1 \mathrm{eV})$, which is difficult to achieve with gas or plasma sources.

Previous surface ionizers designed as ion thrusters have used a sintered $\mathrm{W}$ plug as a diffuser to control the flow of Cs atoms from a rear mounted vapor $\mathrm{Cs}$ reservoir to the front surface ionizer [1]. For the HIF Cs emitter, the sintered iridium "sponge" is both the contact ionizer and the Cs reservoir; the Cs is stored in an ionized state on the large surface area of the porous Ir. We expect a much slower depletion process that is controlled by surface diffusion with essentially immeasurably small vapor pressures.

\section{DESIGN}

\subsection{Ion and Neutral Emission Considerations}

Previously we have used porous $\mathrm{W}$ in the same manner, yielding emission currents of $\mathrm{J} \approx 2 \mathrm{~mA} / \mathrm{cm}^{2}$, but changed from $\mathrm{W}$ to $\mathrm{Ir}$ in the expectation of reduced Cs neutral emission. From the Saha-Langmuir equation, the ion to neutral emission fraction from a clean surface is:

$\frac{v_{i}}{v_{n}}=2 \exp \left[\frac{(\phi-I)}{k T}\right]$

where $\phi$ is the work function of the metal and I is the ionization potential of the adsorbed atom. This ratio is 624 for $\mathrm{Cs}$ on $\mathrm{W}$, and $2 \times 10^{6}$ for Cs on Ir. Additionally, the difference in work functions results in overall emission that is somewhat less for iridium than for $\mathrm{W}$. Therefore, the source needs to operate at a higher temperature $\left(+\sim 100^{\circ} \mathrm{C}\right)$ to achieve the same ion current density. The duty factor of a HIF injector is $2 \times 10^{-4}$, so neutral emission dominates the depletion of Cs on W. We believe Ir, being a noble metal, is fairly clean at the 1000-1200 ${ }^{\circ} \mathrm{C}$ operating temperatures, and therefore less prone to adverse chemical effects than W. The experimentally observed longer emission lifetime (50 hrs vs. $15 \mathrm{hrs})$ and higher current density $\left(8 \mathrm{~mA} / \mathrm{cm}^{2}\right.$ vs. 2 $\mathrm{mA} / \mathrm{cm}^{2}$ ) for the Ir compared to the W seem to confirm the above expectations.

In the $\mathrm{W}$ sponge, diffusion models developed by Langmuir and Taylor [2] predict a diffusion time of $\tau \approx$ 200 seconds compared to an emission lifetime or Cs depletion time of $\sim 15$ hours. For this system, at any instant the surface coverage $\theta$ is constant within the volume of sintered material. In the case of Ir, Cs ions are more tightly bound to the surface, and there is some indication that we may have non-uniform concentrations within the sponge. Thus, we are looking for the time dependence of the depletion, essentially a $t^{-1 / 2}$ decay instead of $\mathrm{e}^{-t / \tau}$, at different temperatures. In the porous $\mathrm{W}$, the initial doping of the $\mathrm{Cs}$ is based on achieving a small $(\theta \sim 3 \%)$ surface coverage. In the Ir sponges, if diffusion from the volume of the sponge to the emitting surface is the limiting process, we may be able to increase the surface coverage in the interior region and gain additional life.

\footnotetext{
*Work supported by the Director, Office of Energy Research, Office of Fusion Energy, U.S. DOE, under contract No. DE-AC03-76SF00098.

\# email: samaclaren@lbl.gov
} 


\subsection{Re-supply of Cs}

It is desirable to have some means of closely controlling $\theta$ to get high current. By using the large surface areas of porous sintered metals as a reservoir the time over which the emission of ions falls below the space charge limit of interest $\left(8 \mathrm{~mA} / \mathrm{cm}^{2}\right)$ can be several days.

Various possible schemes may be used to re-supply Cs atoms to the source, ranging from a continuous Cs vapor feed to a pulsed vapor feed to an infrequent application of a Cs-containing compound. We used a spark source for several years to supply Cs for a 1-Ampere beam on every pulse, and more recently we have used a $\mathrm{Cs}_{2} \mathrm{CO}_{3}$ solution that we apply every few days onto the porous $\mathrm{W}$ emitters with a syringe.

Two methods are currently being considered for resupplying the $\mathrm{Ir}$ emitter with $\mathrm{Cs}$ under vacuum. One approach uses a liquid reservoir of $\mathrm{Cs}_{2} \mathrm{CO}_{3}$ solution, a small amount of which would be sprayed at the cold source under vacuum through a retractable nozzle. A short burst could deliver enough cesium atoms to supply a large driver-scale source for a full day. Another method uses a small array of commercially available alkali dispensers that would be remotely positioned in front of the emitter similar to the nozzle. The dispensers are activated by $\approx 7 \mathrm{~A}$ of current and emit of Cs atoms; this method has been successfully tested. It is appropriate for small sources for scaled experiments, but the storage capacity of the dispensers could not re-supply a HIF driver scale source more than a few times. We are currently investigating another long-term vapor delivery option using a directed nozzle and a much larger reservoir of Cs compound.

\subsection{Effects on High Voltage}

One of the main motivations for using the minimum amount of Cs and for using it efficiently is that even a small amount of Cs on insulators and high voltage electrodes tends to lower their breakdown strength. We presently run a $200 \mathrm{kV}$ Cs beam combining experiment [3], a $160 \mathrm{kV}$ scaled final focusing experiment [4] and a 2 MV $\mathrm{K}$ injector without noticeable problems from contamination, but have had such problems in previous experiments. This is one of the leading concerns about using contact ionization sources in injectors for heavy ion fusion.

\subsection{Source, Filament and Heat Shield}

The heat shield assembly was built with an emphasis on achieving higher temperatures $\left(>1150{ }^{\circ} \mathrm{C}\right)$ for higher current densities with an Ir emitter while still maintaining good thermal efficiency. The filament is freestanding in a Mo chamber, in contrast to our previous potted filaments, or filaments in contact with alumina insulators.

Figure 1 shows a CAD cutaway view of the complete source, including the sintered iridium emitter, the filament, the heat shields, and the carbon Pierce electrode.
The conductive heat path from the Mo cup enclosing the filament is via the reentrant path of three concentric thin (0.005") molybdenum cylinders to reach the back plate of the assembly which would act as a thermal sink. Radiation to the sides and rear of the filament encounters many layers of highly reflective shielding.

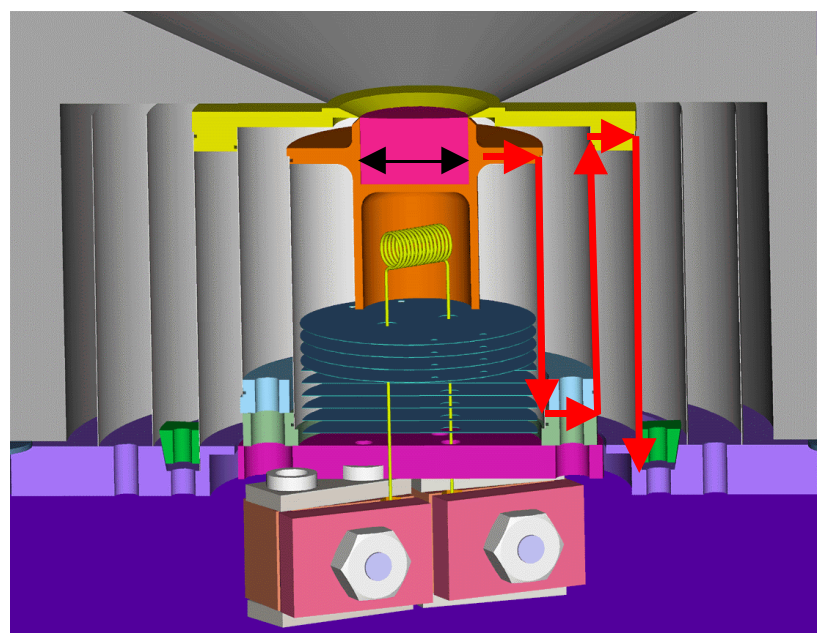

Figure 1: CAD cutaway view of the source, filament, heat shield assembly and the conical Pierce electrode. The Ir emitter diameter is $1.0 \mathrm{~cm}$ (black arrows). The red arrows indicate the conductive path from the filament housing to the rear support flange.

The curved (11 cm radius) emitting surface is the end of a sintered iridium cylinder, $1 \mathrm{~cm}$ in diameter and $1 \mathrm{~cm}$ long. The emitter was then finished to size with a diamond grinder, including grinding the spherical surface. This surface was then etched using electric discharge machining to recover the porous nature of the sintered material near the emitting surface. This allows Cs atoms attached to the internal surfaces of the sintered metal to migrate forward and repopulate the emitting area. Figure 2 shows an electron microscope image of the porous emitting surface.

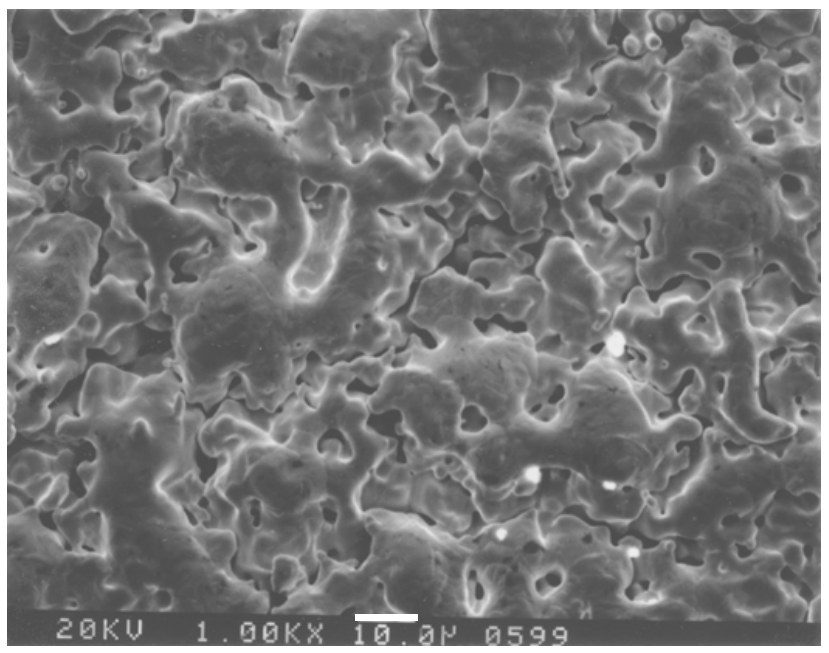

Figure 2: Electron microscope image of the sintered Ir. The line indicates $10 \mu$ on the surface. 
Another important detail of the design is the molybdenum ring that serves as an intermediate stage between the emitter edge and the edge of the carbon Pierce electrode. It is positioned <0.005" from the emitting surface, but does not make thermal contact. Because the properties of the extracted ion beam are very sensitive to the geometry in the immediate vicinity of the emitting surface, a Mo intermediate piece was used so that the inner surface could be machined to a "knife edge". This positions the Pierce angle of the electrode as close as possible to the emitter edge. The less precise edge of the graphite electrode is then no longer a part of the critical region adjacent to the emitter.

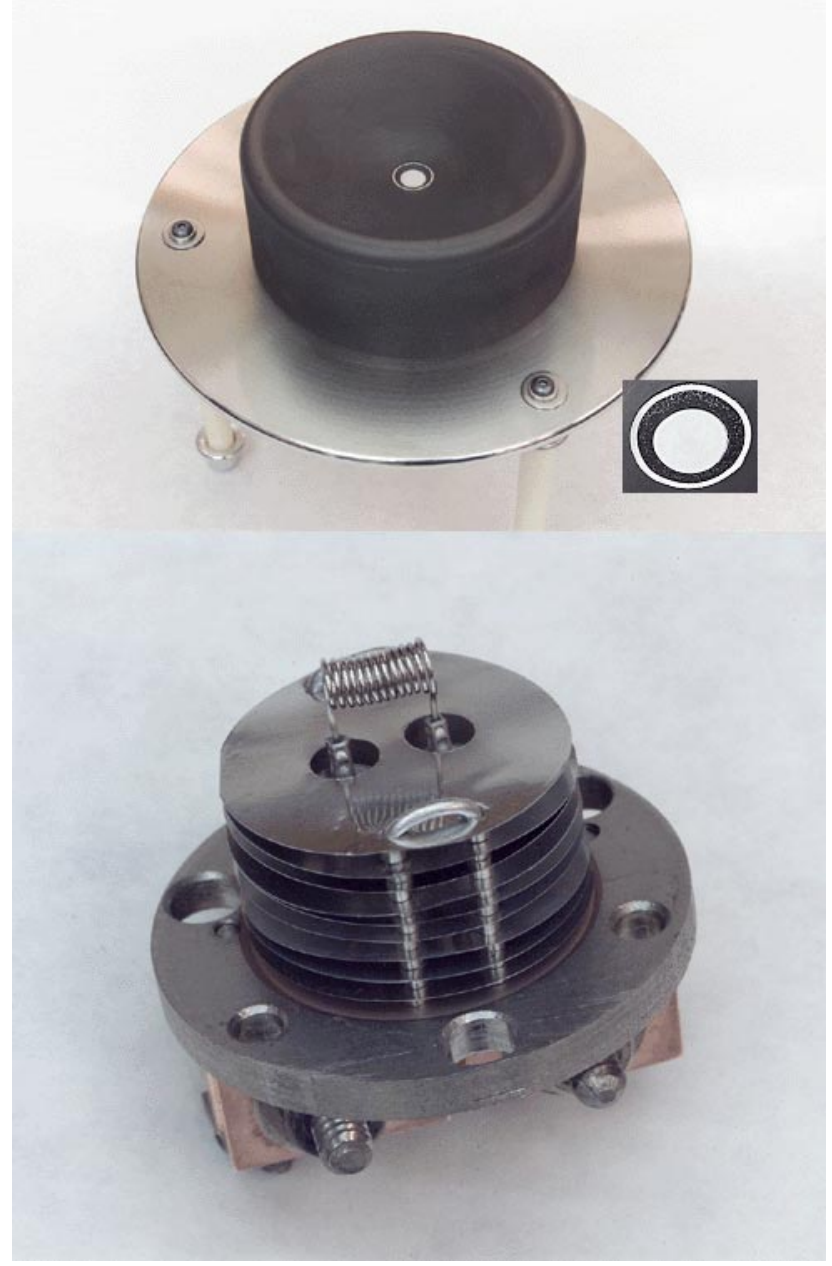

Figure 3: Source and filament after $\sim 100$ hours of use.

\section{PERFORMANCE AND FUTURE PLANS}

The thermal performance of the heat shield assembly is quite good. The emitter surface was raised to a temperature of $1140^{\circ} \mathrm{C}$ using a filament power of 40 watts, and the source was operated at an emitter temperature as high as $1225{ }^{\circ} \mathrm{C}$ using 52 watts. Upon disassembly, there was no visible degradation of the filament or damage to any of the heat shield package components (See fig. 3). The pyrometer used for all measurements was calibrated against thermocouple measurements made of an identical piece of sintered Ir over a temperature range of 900 to $1150^{\circ} \mathrm{C}$.

In fig. 4 we compare our observations to some previous measurements. The data from Wilson [6] are criticalcurrent measurements. It appears that $\mathrm{Cs}$ on Ir will give sufficiently high current density for HIF applications at an acceptable operating temperature.

We observed a space charge limited current of $9 \mathrm{~mA} / \mathrm{cm}^{2}$ for $>50 \mathrm{hrs}$ of continuous running without need to resupply the Ir with Cs. This represents a significant increase in lifetime over our experience with similar sized porous W emitters [3] where the current density was considerably lower $\left(\approx 2 \mathrm{~mA} / \mathrm{cm}^{2}\right)$.

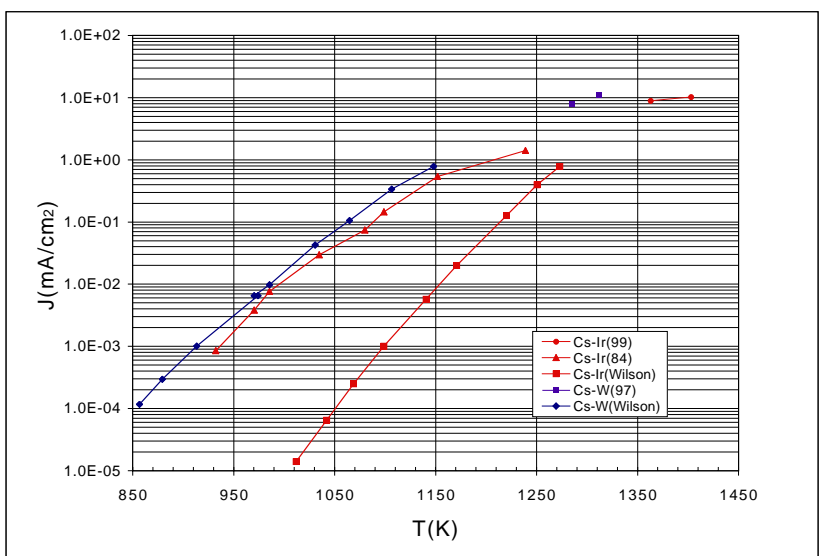

Figure 3: Current density vs. T.

The source will be used in a scaled HIF final focusing experiment [4] where the emittance and beam uniformity will be measured. This design may be implemented in a driver-scale injector [5].

\section{ACKNOWLEDGEMENTS}

The authors gratefully acknowledge the technical support of Mr. Tak Katayanagi.

\section{REFERENCES}

[1] A. Theodore Forrester, "Large Ion Beams", Wiley (1988) 242.

[2] John B. Taylor and Irving Langmuir, Phys. Rev 44, 6 (1933) 423.

[3] P. A. Seidl et al., NIM A415 (1988) 243.

[4] S. MacLaren et al., "Preliminary Results from a Scaled Final Focus Experiment for Heavy Ion Inertial Fusion", these proceedings.

[5] J. Kwan, "A Multiple-Beam Injector for Heavy Ion Fusion", these proceedings.

[6] R. G. Wilson, IEEE $9^{\text {th }}$ Ann. Symp. on Electron, Ion, and Laser Beam Technology, IEEE\# F-79, (1967) 22 